\title{
THE THREE GAP THEOREM (STEINHAUS CONJECTURE)
}

\author{
TONY VAN RAVENSTEIN
}

(Received 18 November 1986; revised 6 August 1987)

Communicated by J. H. Loxton

\begin{abstract}
This paper is concerned with the distribution of $N$ points placed consecutively around the circle by an angle of $\alpha$. We offer a new proof of the Steinhaus Conjecture which states that, for all irrational $\alpha$ and all $N$, the points partition the circle into arcs or gaps of at least two, and at most three, different lengths. We then investigate the partitioning of a gap as more points are included on the circle. The analysis leads to an interesting geometrical interpretation of the simple continued fraction expansion of $\alpha$.
\end{abstract}

1980 Mathematics subject classification (Amer. Math. Soc.) (1985 Revision): 10 F 40, 10 F 05.

\section{Introduction}

The Three Gap Theorem was originally a conjecture of $\mathrm{H}$. Steinhaus: proofs were subsequently offered by various authors $([2],[8-12])$. Consider the sequence $\omega_{N}(\alpha)=(\{n \alpha\}=n \alpha \bmod 1), n=0,1,2, \ldots, N-1$, and let $\{n \alpha\}$ designate the point on the circle of unit circumference lying a clockwise circumferential distance of $\{n \alpha\}$ from the origin. $(\{x\}$ denotes the fractional part of $x$, and $[x]$ the integer part such that $x=[x]+\{x\}$ and $y \bmod x=y-x[y / x]=x\{y / x\}$.) The theorem states that the circumference is then partitioned into arcs or gaps of at most three, and at least two, different lengths for any irrational $\alpha$ (and any $N$ ). This theorem does not appear to be well known and our purpose here is to review the

(C) 1988 Australian Mathematical Society $0263-6115 / 88 \$ A 2.00+0.00$ 
result and offer a new proof. In particular, we formulate transformation rules for the partitioning of a gap.

The theorem is related to the arrangement of elements of $\omega_{N}(\alpha)$ into ascending order of magnitude. Let $\left(\left\{u_{j}(N) \alpha\right\}\right), j=1,2, \ldots, N$, be that ordered sequence. That is, $\left\{u_{1}(N), u_{2}(N), \ldots, u_{N}(N)\right\}=\{0,1, \ldots, N-1\}$ where $\left\{u_{j}(N) \alpha\right\}<\left\{u_{j+1}(N) \alpha\right\}$. In Section 2, we determine the recurrence relation which allows one to determine this ordered sequence. Section 3 obtains values involved in this expression in terms related to the simple continued fraction expansion (C.F.) of $\alpha$. From this result we present an interesting geometrical interpretation of the C.F. of $\alpha$ by investigating (in Section 4) the change in gap structure induced by the addition of extra points to the circle. Results concerning rational approximations to $\alpha$ are derived from Section 3 and presented in the appendix.

All results hold as well for rational $\alpha$, say $\alpha=p / q$ in lowest terms, where it is always assumed that $N<q$. (If $N=q$, the circle is partitioned into $q$ gaps of length $1 / q$.)

\section{The recurrence relation}

We call $u_{j}(N)=u_{j}$ the point which lies on the circle of unit circumference, a clockwise distance of $\left\{u_{j} \alpha\right\}$ from the origin. The operators Pre and Suc are defined such that $u_{j}=\operatorname{Pre}\left(u_{j+1}\right)=\operatorname{Suc}\left(u_{j-1}\right), 2 \leq j \leq N$. We let $d_{i, j}(N)=d_{i, j}$ denote the shortest clockwise distance from point $i$ to point $j$. It is clear that

$$
d_{i, j}=\{\{j \alpha\}-\{i \alpha\}\}=\{(j-i) \alpha\}, \quad 0 \leq i, j<N .
$$

We do not always measure distance in the clockwise direction-if we say that point $i$ is closest to point $j$ then we mean that no other point is closer to point $j$ in the clockwise or anti-clockwise direction.

Consider those points located a distance $\{q \alpha\}$ from a point $j$ where $0<q<N$ (and thus, $0<j<N-q$ ). Of these points, $j+u_{2}$ is the successor of point $j$ since $\left\{u_{2} \alpha\right\}$ is the smallest of all possible distances, $\{q \alpha\}$. That is, using (1), for $0<j<N-q$,

$$
\min _{0<q<N} d_{j, j+q}=\min _{0<q<N} d_{0, q}=d_{0, u_{2}}=d_{k, k+u_{2}}, \quad 0 \leq k<N-u_{2} .
$$

Similarly, for $q \leq j<N$,

$$
\min _{0<q<N} d_{j, j-q}=\min _{0<q<N} d_{q, 0}=d_{u_{N}, 0}=d_{k, k-u_{N}}, \quad u_{N} \leq k<N .
$$

This allows us to state the following (two gap) case. 
LEMMA 2.1. For $N=u_{2}+u_{N}$,

$$
u_{j}=\left((j-1) u_{2}\right) \bmod N, \quad j=1,2, \ldots, N \text {. }
$$

ProOF. From (2) and (3), for this value of $N$,

$$
\operatorname{Suc}(m)-m= \begin{cases}u_{2}, & 0 \leq m<u_{N} \\ -u_{N}, & u_{N} \leq m<N\end{cases}
$$

or

$$
\operatorname{Suc}(m)-m= \begin{cases}u_{2}, & 0 \leq m<N-u_{2}, \\ u_{2}-N, & N-u_{2} \leq m<N .\end{cases}
$$

Hence, $u_{j+1}=\left(u_{j}+u_{2}\right) \bmod N, j=1,2, \ldots, N$, from which the lemma follows.

Equation (4) shows that the circle of $u_{2}+u_{N}$ points is partitioned into $u_{N}$ gaps of length $\left\|u_{2} \alpha\right\|$ and $u_{2}$ gaps of length $\left\|u_{N} \alpha\right\|$, where $\|x\|=\min (\{x\}, 1-\{x\})=$ $|x-[x+1 / 2]|$, the difference between $x$ and its nearest integer.

The following is a proof of the conjecture of Steinhuas.

THEOREM 2.2 .

$$
\operatorname{Suc}(m)-m= \begin{cases}u_{2}, & 0 \leq m<N-u_{2} \\ u_{2}-u_{N}, & N-u_{2} \leq m<u_{N} \\ -u_{N}, & u_{N} \leq m<N\end{cases}
$$

PROOF. Firstly observe that $\max \left(u_{2}, u_{N}\right)<N \leq u_{2}+u_{N}$. (Clearly, $N>$ $\max \left(u_{2}, u_{N}\right)$. Also, if $N>u_{2}+u_{N}$, then $u_{2}+u_{N}$ would lie closer to the origin than one of $u_{2}$ or $u_{N}$, thus contradicting their definition.)

From the sequence of $M=u_{2}+u_{N}$ points, remove $i=M-N$ points; $u_{2}+u_{N}-1, u_{2}+u_{N}-2, \ldots, u_{2}+u_{N}-i$. That is, remove the successors of points $r ; r=u_{N}-i, u_{N}-i+1, \ldots, u_{N}-1$, so that the original $N$ points are left on the circle. Note that $u_{2}(M)=u_{2}(N), u_{M}(M)=u_{N}(N)$, and so $M=u_{2}+u_{M}$. Then from (4),

$$
\begin{aligned}
& \operatorname{Suc}(r)=r+u_{2}, \\
& \operatorname{Suc}(\operatorname{Suc}(r))=r+u_{2}-u_{N}
\end{aligned}
$$

where

$$
\begin{aligned}
r & =u_{N}-i, u_{N}-i+1, \ldots, u_{N}-1 \\
& =N-u_{2}, N-u_{2}+1, \ldots, u_{N}-1 .
\end{aligned}
$$

Thus, on the circle of $N$ points, the successor of point $r$ is $r+u_{2}-u_{N}$ where $r=$ $N-u_{2}, N-u_{2}+1, \ldots, u_{N}-1$. Combining this with (4) proves the theorem.

The theorem shows that the circle of $N$ points is partitioned into $N-u_{2}$ gaps of length $\left\|u_{2} \alpha\right\|, N-u_{N}$ gaps of length $\left\|u_{N} \alpha\right\|$ and $u_{2}+u_{N}-N$ gaps of length $\left\|u_{2} \alpha\right\|+\left\|u_{N} \alpha\right\|$. 


\section{The predecessor and successor of the origin}

We determine $u_{2}$ and $u_{N}$ in terms of the C.F. of $\alpha$. First, we introduce some notation from the theory of continued fractions.

We define the C.F. of $\alpha$ by the following algorithm: we write $t_{0}=\alpha$ and define (for $n=0,1,2, \ldots$ ),

$$
a_{n}=\left[t_{n}\right], \quad t_{n+1}=\frac{1}{\left\{t_{n}\right\}} .
$$

In this way we express the C.F. of $\alpha$ by

$$
\begin{aligned}
\alpha & =a_{0}+\frac{1}{a_{1}+\frac{1}{a_{2}+\frac{1}{a_{3}+\cdots}}} \\
& =\left\{a_{0} ; a_{1}, a_{2}, a_{3}, \cdots\right\} .
\end{aligned}
$$

It is evident that $t_{n}$ is the $n$th tail of our C.F. such that

$$
t_{n}=\left\{a_{n} ; a_{n+1}, \ldots\right\} \text {, }
$$

and

$$
\alpha=\left\{a_{0} ; a_{1}, a_{2}, \ldots, a_{n-1}, t_{n}\right\} .
$$

We say that $\alpha$ is equivalent to $\beta$ if we can find some tail in $\alpha$ which is equal to some tail in $\beta$.

Note that the algorithm terminates (so that the number of terms in the continued fraction expansion is finite) if and only if $\alpha$ is rational.

Partial convergents are defined by the (irreducible) fractions

$$
\frac{p_{n, i}}{q_{n, i}}=\frac{p_{n-2}+i p_{n-1}}{q_{n-2}+i q_{n-1}}=\left\{a_{0} ; a_{1}, a_{2}, \ldots, a_{n-1}, i\right\}, \quad i=1,2, \ldots, a_{n},
$$

where

$$
\frac{p_{n, a_{n}}}{q_{n, a_{n}}}=\frac{p_{n}}{q_{n}}, \quad p_{-2}=q_{-1}=0, \quad q_{-2}=p_{-1}=1
$$

We call $p_{n} / q_{n}$ a total convergent to $\alpha$.

We note the following results which may be easily proved. (See, for example, Khintchine [4].)

$$
\begin{gathered}
p_{n-1} q_{n, i}-q_{n-1} p_{n, i}=(-1)^{n}, \\
\left\|q_{n, i} \alpha\right\|=\left\|q_{n-2} \alpha\right\|-i\left\|q_{n-1} \alpha\right\|, \\
q_{n} \alpha-p_{n}=\frac{(-1)^{n}}{t_{n+1} q_{n}+q_{n-1}} .
\end{gathered}
$$

The following result comes from Diophantine approximation theory and for the proof we refer the reader to Khintchine [4]. This lemma shows that point $q_{n-1}$ is closest to the origin for $q_{n-1}<N \leq q_{n, i}, i=1,2, \ldots, a_{n}(n \geq 2)$. 
LEMMA 3.1 .

$$
\min _{0<q<q_{n, i}}\|q \alpha\|=\left\|q_{n-1} \alpha\right\|, \quad i=1,2, \ldots, a_{n}(n \geq 2) .
$$

The following lemma proves that, for $N$ in the same interval, point $q_{n, i-1}$ is the next closest point on the opposite side of the origin to point $q_{n-1}$.

LEMMA 3.2.

$$
\min _{0<q<q_{n, i}}\|q \alpha\|=\left\|q_{n, i-1} \alpha\right\|, \quad i=1,2, \ldots, a_{n}(n \geq 2),
$$

where $k$ is integer so that $0<k q_{n-1}<q_{n, i}$ or $k<q_{n-2} / q_{n-1}+i$ or $k \leq i \leq a_{n}$.

ProOF. We emphasise that $q$ is integer, lies between 0 and $q_{n, i}$, and may not be a multiple of $q_{n-1}$. We write

$$
q=\mu q_{n-1}+\nu q_{n, i}, \quad p=\mu p_{n-1}+\nu p_{n, i} .
$$

Solving for $\mu$ and $\nu$ using (6) yields the integer solution:

$$
\mu=(-1)^{n}\left(p q_{n, i}-q p_{n, i}\right), \quad \nu=(-1)^{n-1}\left(p q_{n-1}-q p_{n-1}\right) .
$$

Neither $\mu$ nor $\nu$ may equal 0 (if $\mu=0$, then $q=\nu q_{n, i}$, while if $\nu=0, q=\mu q_{n-1}-$ two obvious contradictions). Note that

$$
q \alpha-p=\mu\left(q_{n-1} \alpha-p_{n-1}\right)+\nu\left(q_{n, i} \alpha-p_{n, i}\right) .
$$

Now, $0<q=\mu q_{n-1}+\nu q_{n, i}<q_{n, i}$. This shows that $\mu$ and $\nu$ are of opposite sign. Also, $\left(q_{n-1} \alpha-p_{n-1}\right)$ and $\left(q_{n, i} \alpha-p_{n, i}\right)$ are of opposite sign, which may be deduced using (8). Thus,

$$
\|q \alpha\| \geq\left\|q_{n-1} \alpha\right\|+\left\|q_{n, i} \alpha\right\| .
$$

That is, from (7),

$$
\|q \alpha\| \geq\left\|q_{n, i-1} \alpha\right\| .
$$

Equality occurs when $|\mu|=|\nu|=1$. If $\mu=-\nu=1$, then $q=q_{n-1}-q_{n, i}<0$ (a contradiction), while $-\mu=\nu=1$ implies that $q=-q_{n-1}+q_{n, i}=q_{n, i-1}$. Thus we conclude that

$$
\|q \alpha\|>\left\|q_{n, i-1} \alpha\right\|, \quad 0<q<q_{n, i}, q \neq k q_{n-1}, \quad k=1,2, \ldots, i .
$$

We mention that the upper bound of $k$ (that is, $i$ ) in Lemma 3.2 may be replaced by $\min \left(i, a_{n}-i+1\right)$.

From Lemmas 3.1 and 3.2, one may deduce the following theorem. The proof which we omit, is fairly routine. 
THEOREM 3.3 .

$$
u_{2}=\left\{\begin{array}{ll}
q_{n-1}, & n \text { odd, } \\
q_{n, i-1}, & n \text { even, }
\end{array} \quad u_{N}= \begin{cases}q_{n, i-1}, & n \text { odd, } \\
q_{n-1}, & n \text { even, }\end{cases}\right.
$$

where $q_{n, i-1}<N \leq q_{n, i}, 2 \leq i \leq a_{n}(n \geq 2)$.

For $q_{n-1}<N \leq q_{n, 1}(n \geq 2)$,

$$
u_{2}=\left\{\begin{array}{ll}
q_{n-1}, & n \text { odd }, \\
q_{n-2}, & n \text { even, }
\end{array} \quad u_{N}= \begin{cases}q_{n-2}, & n \text { odd }, \\
q_{n-1}, & n \text { even. }\end{cases}\right.
$$

For $N \leq q_{1}, u_{j}=j-1, j=1,2, \ldots, N$.

\section{Gap structure}

The following describes the change in gap structure induced by the transition from a circle of $q_{n-1}$ or $q_{n, i}$ gaps to one of $q_{n, 1}$ or $q_{n, i+1}$ gaps respectively ( $i=$ $\left.1,2, \ldots, a_{n}-1\right)$. The analysis provides an interesting geometrical interpretation of the C.F. of $\alpha$.

Suppose that the circle is partitioned into gaps of only two different lengths which we describe as large and small. We label a large gap $l$ and call a small gap $s$. Let

$$
\begin{gathered}
\Phi_{n, i}=\phi_{n, i}^{1} \phi_{n, i}^{2} \ldots \phi_{n, i}^{q_{n, i}}, \\
\Phi_{n, a_{n}}=\Phi_{n},
\end{gathered}
$$

where $i=1,2, \ldots, a_{n}$, denote the string of gap types for $N=q_{n, i}, i=1,2, \ldots, a_{n}$ $(n \geq 1)$ ordered clockwise around the circle so that $\phi_{n, i}^{j}$ denotes the gap type (either $s$ or $l$ ) formed by the points $u_{j}\left(q_{n, i}\right)$ and $u_{j+1}\left(q_{n, i}\right)$. Assume that $\Phi_{0}=s$.

Define $P_{n, i}$ such that

$$
P_{n, i}(l)= \begin{cases}s l, & n \text { odd } \\ l s, & n \text { even }\end{cases}
$$

where $i=1,2, \ldots, a_{n}$,

$$
\begin{aligned}
& P_{n, 1}(s)=l, \\
& P_{n, i}(s)=s, \quad i=2,3, \ldots, a_{n} .
\end{aligned}
$$

Assume that $P_{n, i}$ is a homomorphism such that, for instance, $P_{n, i}(s l)=$ $P_{n, i}(s) P_{n, i}(l)$.

THEOREM 4.1.

$$
\begin{aligned}
\Phi_{n, 1} & =P_{n, 1}\left(\Phi_{n-1}\right), \\
\Phi_{n, i+1} & =P_{n, i+1}\left(\Phi_{n, i}\right), \quad i=1,2, \ldots, a_{n}-1 .
\end{aligned}
$$


ProOF. Suppose that $N=u_{2}+u_{N}=q_{n-1}$, where $n$ is odd so that there are $u_{N}$ large gaps of length $\left\|u_{2} \alpha\right\|$ and $u_{2}$ small gaps of length $\left\|u_{N} \alpha\right\|$. Let $a=u_{2}$.

Now place point $q_{n-1}$ on the circle. From Theorem 3.3 it enters the large gap succeeding the origin and becomes the new Suc(0), that is $u_{2}$. From Lemma 3.1 this point is closest to 0 and thus the gap $l$ is transformed into $s l$. The next point, $q_{n-1}+1$, enters the large gap succeeding point 1 , transforming this $l$ into $s l$. As $N$ is increased to $q_{n, 1}$ all new points enter in succession the large gaps which succeed points $m$ where $m=1,2, \ldots, u_{N}-1$ : thus all the small gaps are of length $\left\|u_{2} \alpha\right\|$ and all the (new) large gaps are of length $\|a \alpha\|-\left\|u_{2} \alpha\right\|=\left\|u_{N} \alpha\right\|=\left\|u_{M} \alpha\right\|$ where $M=q_{n-1}$. Hence the small gaps present when $N=q_{n-1}$ are now labelled as large and $\Phi_{n, 1}=P_{n, 1}\left(\Phi_{n-1}\right)$.

If $a_{n}>1$ the next point $q_{n, 1}=q_{n-1}+q_{n-2}$ becomes the new Pre(0) and, since $q_{n-1}$ is still the closest point to the origin, it transforms the large gap $l$ it divides into $s l$. As $N$ is increased to $q_{n, 2}$ these new points successively divide the large gaps succeeding points $m$ where $m=1,2, \ldots, u_{2}-1$. Thus all the large gaps present when $N=q_{n, 1}$ are transformed into $s l$, where the length of the small gap is equal to $\left\|u_{2} \alpha\right\|$. Thus, each small gap remains undivided and retains its label $s$ and hence $\Phi_{n, 2}=P_{n, 2}\left(\Phi_{n, 1}\right)$. Similar statements may be made for the transition from $N=q_{n, i}$ to $N=q_{n, i+1}-1\left(i=2,3, \ldots, a_{n}-1\right)$.

The proof for even $n$ is omitted since it follows in a like manner.

Theorem 4.1 leads us to the following observation and geometrical interpretation of the C.F. of $\alpha$ : each large gap present at $q_{n-1}$ points $(n \geq 2)$ is partitioned into $a_{n}$ small gaps of length $\left\|q_{n-1} \alpha\right\|$ and a new large gap of length $\left\|q_{n-1} \alpha\right\|+\left\|q_{n} \alpha\right\|$ as we go to $q_{n}-1$ points. If we pretend that each of the large gaps present when $N=q_{n-1}+q_{n-2}$ are circles of unit circumference, then as $N$ is increased they appear as if they are being divided by an angle of $1 / t_{n}$ for odd $n$ and $1-1 / t_{n}$ for even $n$.

Each point divides some gap forming two new gaps-we interpret this event as the death of an old gap and the simultaneous birth of two new gaps and thus define the age of the gap with endpoints $u_{j}, u_{j+1}$ to be

$$
a_{j}(N)=N-1-\max \left(u_{j}, u_{j+1}\right) .
$$

Proposition 4.2. Suppose we have placed $N-1$ points and are to place point $N-1$. Then, point $N-1$ divides that gap which is the oldest of the largest gaps. The age of this gap is always $\min \left(u_{2}(N), u_{N}(N)\right)-1$. (Note that $u_{2}$ and $u_{N}$ relate to points adjacent to the origin after the point $N-1$ has been placed.)

ProOF. From Theorem 2.2, each additional point $N-1$ divides a large gap, since $\operatorname{Suc}(N-1)-\operatorname{Pre}(N-1)=u_{2}-u_{N}$. It now remains to show that this gap is the oldest. From Theorem 2.2, each point $N-1$ divides the gap which is of 
age

$$
\begin{aligned}
N-2-\max \left(N-1-u_{2}, N-1-u_{N}\right) & =N-2-\left(N-1+\max \left(-u_{2},-u_{N}\right)\right), \\
& =\min \left(u_{2}, u_{N}\right)-1 .
\end{aligned}
$$

If this is not the oldest of the large gaps then the gap, say, with endpoints $a$ and $b$ which we will call $g_{a, b}$ is older. Since $g_{a, b}$ is a large gap it follows that $b=a+u_{2}-u_{N}$. Without loss of generality assume that $u_{2}>u_{N}$. Then $g_{a, b}$ is of age $N-2-\max (a, b)=N-2-a-u_{2}+u_{N}$. It then follows that $N-2-a-u_{2}+u_{N}>u_{N}-1$ or $N-1-a>u_{2}$. From Theorem 2.2, if $b-a=u_{2}-u_{N}$, then $N-u_{2} \leq a<u_{N}$. Thus, $N-1-u_{N}<N-1-a \leq u_{2}-1$, which contradicts the above and hence completes the proof.

\section{Discussion}

We conclude with a brief survey (using our notation) of the different approaches adopted by various authors to prove the Steinhaus Conjecture.

Our approach was to first describe the case (Lemma 2.1) where the circle is partitioned into gaps of just two different lengths. This was achieved by applying the identities (2) and (3). Points were then carefully removed and the situation analysed to determine Theorem 2.2, a statement of the Three Gap Theorem. Neighbouring points to the origin, $u_{2}$ and $u_{N}$, were derived in terms of the C.F. of $\alpha$ via Lemmas 3.1 and 3.2 which (as shown in the appendix) are concerned with 'best' and 'second best' rational approximations to $\alpha$.

One of the first proofs was offered by Świerczkowski [11] who determined the recurrence relation after showing that the first point to replace either $\operatorname{Suc}(0)$ or $\operatorname{Pre}(0)$ is the point $\operatorname{Suc}(0)+\operatorname{Pre}(0)$. Świerczkowski did not relate any of his results to continued fractions. As a corollary he proved a conjecture of J. Oderfeld, determining the gap sizes for the case $\alpha=(\sqrt{5}-1) / 2$ by using this result to show inductively that $\operatorname{Suc}(0)$ and $\operatorname{Pre}(0)$ are consecutive Fibonacci numbers.

The proof offered by Sós $[9,10]$ is similar in approach to that of Świerckowski. In [10] Sós identified the points $\operatorname{Suc}(0)$ and $\operatorname{Pre}(0)$ with the denominators of convergents to $\alpha$. She also showed that if the terms in the C.F. of $\alpha$ are unbounded, then

$$
\begin{aligned}
& \liminf _{N \rightarrow \infty} N h_{N}=0, \quad \liminf _{N \rightarrow \infty} N H_{N}=1, \\
& \limsup _{N \rightarrow \infty} N h_{N}=1, \quad \limsup _{N \rightarrow \infty} N H_{N}=\infty,
\end{aligned}
$$

where $H_{N}$ and $h_{N}$ denote, respectively, the lengths of the largest and smallest gap belonging to a circle of $N+1$ points. These results were also conjectured by Steinhaus and first proved by Hartman [3] using the theory of continued fractions. 
Surányi [12] enclosed $\alpha$ in an interval whose endpoints are consecutive members of a Farey sequence of certain order. That is, he supposed that $A / a<$ $\alpha<B / b$ where $a B-b A=1$ and $A / a, B / b \in F_{N} . F_{N}$ is the Farey sequence of order $N$ (the ordered sequence of irreducible fractions in the unit interval with denominators not greater than $N$ ) where $\max (a, b) \leq N \leq a+b-1$. He then deduced that $a=u_{2}$ and $b=u_{N}$ and that $U_{N}(\alpha)=U_{N}(A / a)$. This result was then used to verify Theorem 2.2 .

The proof offered by Halton [2] involves a description of the dynamics of the gap division with reference to quantities derived from the C.F. of $\alpha$. Halton, however, did not concern himself with ordering the points.

Salter [8] approached the problem by first deducing Theorem 2.2. From this relation it is readily shown that $u_{N}\left\|u_{2} \alpha\right\|+u_{2}\left\|u_{N} \alpha\right\|=1$. To find $u_{2}$ and $u_{N}$ this equation was solved subject to the constraint $A / u_{2}<\alpha<B / u_{N}$ where $A$ and $B$ are the nearest integers to $u_{2} \alpha$ and $u_{N} \alpha$ respectively. Thus Slater was able to express $u_{2}$ and $u_{N}$ in terms of the C.F. of $\alpha$. Slater also considered the problem of determining the relationship between the successive integer values of $j$ for which $\{j \alpha\}<\Phi$ where $0<\Phi<1$. He showed that the 'gaps' between the successive $j$ may take on at most three different values, one being the sum of the other two. This problem was originally discussed in [7].

\section{Note}

It has been pointed out by a referee that Chung and Graham [1] have generalised the Three Gap Theorem. R. L. Graham [5] first conjectured that the $d$ sets of points $\left\{n_{i} \alpha+\beta_{i}\right\}, 0 \leq n_{i}<N_{i}, 1 \leq i \leq d\left(\beta_{1}=0\right)$ partition the circle of unit circumference into gaps of at most $3 d$ different lengths. The proof offered by Chung and Graham is somewhat involved-after its publication a simpler (2-page) proof was produced by Liang [6].

\section{Appendix. Rational approximations to $\alpha$}

In this appendix we interpret Lemmas 3.1 and 3.2 in terms of Diophantine approximation theory. As defined in Khintchine [4], $a / b$ is a best approximation (of the second kind) to $\alpha(B A 2$ to $\alpha$ ) if

$$
\min _{0<q \leq b}\|q \alpha\|=\|b \alpha\|
$$

where $q$ is integer. (Note that $a=[b \alpha+1 / 2]$ and the minimum is unique.)

Lemma 3.1 then expresses the fact that the total convergents to $\alpha$ provide the unique sequence of $B A 2$ 's to $\alpha$. 
We define $c / d$ to be the second best approximation (of the second kind) to $\alpha$ $(B A 22$ to $\alpha)$ if

$$
\min _{\substack{0<q \leq d \\ p / q \neq a / b}}\|q \alpha\|=\|d \alpha\|,
$$

where $c=[d \alpha+1 / 2]$. That is, if we exclude from consideration the $B A 2$ to $\alpha$ $(a / b)$, then $c / d$ is the remaining best approximation. From Lemma 3.1 it is clear that $a / b=p_{n} / q_{n}$, where $n=\max \left\{k: q_{k} \leq d\right\}$. From Lemma 3.2,

$$
\min _{\substack{0<q \leq d \\ p / q \neq a / b}}\|q \alpha\|=\left\|q_{n+1, i} \alpha\right\|, \quad i=0,1,2, \ldots, a_{n+1}-1,
$$

where $i=\max \left\{k: q_{n+1, k} \leq d\right\}$. Thus, to conform with the definition of a $B A 22$, we may replace d by $q_{n+1, i}$ for $i=1,2, \ldots, a_{n+1}-1$. (If $i=0$, the definition of a $B A 22$ is violated since

$$
\min _{0<q \leq d, p_{n} / q_{n} \neq a / b}\|q \alpha\|=\left\|q_{n-1} \alpha\right\| \neq\|d \alpha\|,
$$

as $d \geq q_{n}$. We note that if $d=q_{n}$, we are considering $B A 2$ 's to $\alpha$.)

We conclude that a $B A 22$ to $\alpha$ is necessarily a partial or total convergent to $\alpha\left(c / d\right.$ is a total convergent if $\left.a_{n+1}=1\right)$.

\section{Acknowledgements}

The author is most grateful to Drs. Keith Tognetti and Graham Winley of The University of Wollongong for helpful discussions. As well, a referee is to be commended for improving the clarity of the proof of Theorem 2.2.

\section{References}

[1] F. R. K. Chung and R. L. Graham, 'On the set of distances determined by the union of arithmetic progressions,' Ars Combinatoria 1 (1976), 57-76.

[2] J. H. Halton, 'The distribution of the sequence $\{n \xi\}(n=0,1,2, \ldots)$, Proc. Cambridge Phil. Soc. 71 (1965), 665-670.

[3] S. Hartman, 'Über die Abstände von Punkten $n \xi$ auf der Kreisperipherie,' Ann. Soc. Polon. Math. 25 (1952), 110-114.

[4] A. Y. Khintchine (translated by P. Wynn), Continued fractions (P. Noordhoff Ltd., Groningen, 1963.)

[5] D. E. Knuth, The art of computer programming, Vol. 3, Ex. 6.4.10 (Addison-Wesley, Reading, Mass., 1968.)

[6] F. M. Liang, 'A short proof of the $3 d$ distance theorem,' Discrete Math. 28 (1979), 325-326.

[7] N. B. Slater, 'The distribution of the integers $N$ for which $\{\theta N\}<\phi$,' Proc. Cambridge. Philos. Soc. 46 (1950), 525-543. 
[8] N. B. Slater, 'Gaps and steps for the sequence $n \theta$ mod 1,' Proc. Cambridge Philos. Soc. 73 (1967), 1115-1122.

[9] V. T. Soss, 'On the theory of diophantine approximations. I,' Acta Math. Acad. Sci. Hungar. 8 (1957), 461-472.

[10] V. T. Sós, 'On the distribution $\bmod 1$ of the sequence na,' Ann. Univ. Sci. Budapest. Eötvös Sect. Math. 1 (1958), 127-134.

[11] S. Swierckowski, 'On successive settings of an arc on the circumference of a circle,' Fund. Math. 46 (1958), 187-189.

[12] J. Surányi, 'Über dei Anordnung der Vielfachen einer reelen Zahl mod 1,'Ann. Univ. Sci. Budapest. Eötvös Sect. Math. 1 (1958), 107-111.

Department of Mathematics

The University of Wollongong

Wollongong, N.S.W. 2500

Australia 